\title{
Sprecher/innen der Manchot Graduiertenschule „Wettbewerbsfähigkeit junger Unternehmen“
}

Professorin Dr. Eva Lutz ist Inhaberin des Lehrstuhls für Betriebswirtschaftslehre, insb. Entrepreneurship und Finanzierung an der Heinrich-Heine-Universität Düsseldorf. Gemeinsam mit Professor Dr. Stefan Süß ist sie Sprecherin der Manchot Graduiertenschule „Wettbewerbsfähigkeit junger Unternehmen“. Zudem verantwortet sie die Leitung des Centers for Entrepreneurship Düsseldorf (CEDUS) der Heinrich-Heine-Universität Düsseldorf. Vor ihrer akademischen Karriere arbeitete sie bei L. E. K. Consulting in London, zuletzt als Projektleiterin, in den Bereichen Unternehmensstrategie und Due Diligence. Die Promotion (2005) und Habilitation (2012) erfolgten an der Technischen Universität München. Ihre Schwerpunkte in Forschung und Lehre liegen in den Bereichen Entrepreneurial Finance, Finanzierung von innovativen Unternehmensgründungen und Familienunternehmen, Venture Capital und Private Equity. Weitere Informationen finden sich unter www.ef.hhu.de.

Professor Dr. Stefan Süß ist Inhaber des Lehrstuhls für Betriebswirtschaftslehre, insb. Arbeit, Personal und Organisation an der Heinrich-Heine-Universität Düsseldorf. Gemeinsam mit Professorin Dr. Eva Lutz ist er Sprecher der Manchot Graduiertenschule „Wettbewerbsfähigkeit junger Unternehmen“. Seit Oktober 2018 ist er Dekan der Wirtschaftswissenschaftlichen Fakultät der Heinrich-Heine-Universität Düsseldorf. Die Promotion (2004) und die Habilitation (2009) erfolgten an der FernUniversität in Hagen. Professor Dr. Stefan Süß hat zahlreiche BMBF-geförderte Projekte sowie das NRW-Forschungskolleg zu Online-Partizipation miteingeworben. Aktuelle Forschungsschwerpunkte liegen in verschiedenen Bereichen des Personalmanagements, insbesondere in den Bereichen Arbeitgeberattraktivität, Stressforschung, Flexibilisierung der Arbeit und Personalführung. Weitere Informationen finden sich unter www.orgaperso.hhu.de. 
